\title{
Simulink Implementation of Ka-band Mobile Satellite Channel Comprehensive Model
}

\author{
Chuan $\mathrm{HE}^{1}$, Yecai GUO ${ }^{2, \mathrm{a}^{*}}$, Weijuan $\mathrm{ZHAO}^{2}$ \\ ${ }^{1}$ College of Atmospheric Physics, Nanjing University of Information Science and Technology, \\ Nanjing 210044, China \\ ${ }^{2}$ Jiangsu Collaborative Innovation Center on Atmospheric Environment and Equipment Technology \\ (CICAEET), Nanjing 210044, China \\ ${ }^{{ }^{\star}}$ email: guo-yecai@163.com
}

Keywords: Ka-band; Weather impairments; Comprehensive model; Simulink simulation.

\begin{abstract}
In order to study the propagation characteristics of the Ka-band mobile satellite channel, the influences of weather impairments (rainfall, clouds, etc.), the multipath fading, and shadowing fading on mobile satellite communication are analyzed and taken into consideration simultaneously. After the corresponding weather channel model and two-state channel model of the mobile environment are described based on the channel propagation characteristics, a novel comprehensive model of the Ka-band mobile satellite channel is proposed and simulated by the Simulink simulation software. The simulation results show that the proposed model of Ka band mobile satellite channel can be used to accurately describe the propagation characteristics of Ka-band mobile satellite channel.
\end{abstract}

\section{Introduction}

With the continuous development of communication technology, the use of low frequency communication technology doesn't satisfy the requirement of people. In the field of the modern satellite communication, the Ka-band satellite communication is widely used with the advantages of wide bandwidth, large communication capacity, less interference and so on. However, with the increasing frequency of wireless communication, the effects related to the weather such as rainfall, cloud, and mist are more and more obvious, but weather influence of them on the propagation characteristics can't be ignored.

At present, the research of satellite channel mainly concentrates on the complex mobile environment with various obstacles[1]. For the Ka-band satellite channel, the propagation characteristics of Ka-band fixed satellite channel was researched under the rainfall, but the effect of the mobile environment was neglected[2], whereas the Ka-band mobile satellite channel model, which consideres the rainfall, multipath, and shadowing fading, was a simple static model[3].

To build a Ka-band mobile satellite channel dynamic model in this paper, we first analyze the channel propagation characteristics at Ka-band, then the Ka-band mobile satellite channel comprehensive model is established and simulated by the Simulink simulation software.

\section{Ka-Band Satellite Channel Propagation Characteristics}

In the Ka-band satellite communication, the transmission signal will be affected by the weather conditions and the complex mobile terminal environments such as trees, buildings, and other obstacles. These effects are analyzed as follows:

Weather effect. Ka-band signal propagation is very susceptible to weather conditions such as the rain attenuation and clouds attenuation. According to the ITU-R rain attenuation forecast model[4], the rain attenuation $A_{0.01}=\gamma_{R} L_{E}=k R_{0.01}^{\alpha} L_{E}$, where $R_{0.01}$ is the rain intensity exceeded during $0.01 \%$ of an average year, $\gamma_{R}$ is the rain attenuation rate, $L_{E}$ is an effective path length, $k$ and $\alpha$ are parameters depending on the frequency, polarization, and elevation angle of the earth station.For the 
Ka-band satellite communications, the clouds attenuation is given by $A_{c}=L K_{1} / \sin \theta$ [5], where $\theta\left(10^{\circ} \leq \theta \leq 90^{\circ}\right)$ is the elevation angle, $L$ is the columnar water content, $K_{1}$ is attenuation coefficient, it is relevant to the frequency and the dielectric permittivity of the water.

Multipath and shadowing effect. When the satellite signals pass through the trees, buildings, and other obstacles, the multipath effect is caused by the reflecting, scattering, and diffraction. The received signals are composed of the waveforms from different paths. The Rayleigh distribution can be used to describe the multipath effect and its the probability density function can be expressed as

$$
p_{\text {Ray }}(r)=\frac{r}{\sigma^{2}} \exp \left(-\frac{r^{2}}{2 \sigma^{2}}\right) \quad r \geq 0
$$

where $\sigma^{2}$ is the power of scattering component.

The shadowing effect is caused by the blocking, reflection, and refraction of these obstacles. The received signals will be attenuated by shadowing effects. The Lognormal distribution can be used to describe the shadowing fading and its the probability density function can be expressed as

(2)

$$
p_{L N}(r)=\frac{1}{\sqrt{2 \pi} \sigma_{u} r} \exp \left(-\frac{\left(\ln r-m_{u}\right)^{2}}{2 \sigma_{u}^{2}}\right) \quad r \geq 0
$$

where $\sigma_{u}$ and $m_{u}$ are the standard deviation and mean.

\section{Ka-Band Mobile Satellite Channel Comprehensive Model}

Ka-band mobile satellite channel propagation is effected by weather and the mobile environments. These two effects are considered and the channel models based on them are also established to describe the characteristics of Ka-band mobile satellite channel.

Channel model based on weather effect. At Ka-band, the weather effects mainly include rain attenuation, clouds attenuation and so on. According to the Loo's Ka-band propagation, the envelope of the received signal follows a Gaussian distribution. Its probability density function can be expressed as

$$
p_{w}(r)=\frac{1}{\sqrt{2 \pi} \sigma_{w}} \exp \left(\frac{-\left(r-m_{w}\right)^{2}}{2 \sigma_{w}^{2}}\right)
$$

where $\sigma_{w}$ and $m_{w}$ are the standard deviation and mean.

Land mobile satellite channel model. In the land mobile satellite environments, a single-state model can't be used to accurately describe the propagation characteristics of the satellite channel,whereas the two-state channel model including good-state and bad-state can betterly describe these characteristics[6]. In the good-state, the mobile terminal environment is ideal. The received signal,which is composed of the direct component and the multipath components, isn't affected by shadowing effect. In this case, the channel characteristics can be described as Rician process and the probability density function of the envelope $r$ of the received signal can be expressed as

$$
p_{\text {Rice }}(r)=\frac{r}{\sigma^{2}} \exp \left(-\frac{r^{2}+A^{2}}{2 \sigma^{2}}\right) I_{0}\left(\frac{r A}{\sigma^{2}}\right) \quad r \geq 0
$$

where $\sigma^{2}$ is the average power of multipath scattering, $A$ is the amplitude of the direct component, and $I_{0}(\cdot)$ is the first kind of the modified zero order Bessel function.

In the bad-state, the direct component is blocked completely and the multipath components are affected by the shadowing effect. In this case, the channel characteristics can be described as Suzuki 
model composed of Rayleigh process and Lognormal process. The probability density function of the envelope $r$ of the received signals can be expressed as

$$
p_{\text {Suzuki }}(r)=\frac{r}{\sigma_{0}^{2} \sigma_{u} \sqrt{2 \pi}} \int_{0}^{\infty} \frac{1}{z^{3}} \exp \left(-\frac{(r / z)^{2}}{2 \sigma_{0}^{2}}-\frac{\left(\ln z-m_{u}\right)^{2}}{2 \sigma_{u}{ }^{2}}\right) d z \quad r \geq 0
$$

where $\sigma_{0}^{2}$ is the average power of multipath component, $\sigma_{u}$ and $m_{u}$ are the standard deviation and mean of $\ln (z)$. In two states (good and bad), the probability density function of the envelope $r$ of the received signals are defined as

$$
p_{g}(r)=s_{g} p_{\text {Rice }}(r)+s_{b} p_{\text {Suzuki }}(r)
$$

where $s_{g}$ is the probability of good state, and $s_{b}$ is the probability of bad state.

Ka-band mobile satellite channel comprehensive model. The effects of the weather and the mobile environment are taken into consideration simultaneously. These two effects represented by $w$ and $g$ are assumed to be statistically independent. Therefore, the received signal envelope can be represented as $r=w g$. According to the probability theory knowledge, the probability density function of synthetic signal envelope is written as

$$
p(r)=\int_{0}^{\infty} p(r \mid w) p_{w}(w) d w
$$

where $p(r \mid w)$ is the conditional probability density function of the mobile environment on a certain weather impairments, $p_{w}(w)$ is the probability density function of the weather impairments. When $w$ and $g$ are independent, the $p(r \mid w)$ can be written as

$$
p(r \mid w)=\frac{1}{w} p_{g}\left(\frac{r}{w}\right)
$$

Thus, the probability density function of the envelope $r$ of total received signal can be expressed as

$$
\begin{aligned}
p(r) & =\int_{0}^{\infty} \frac{1}{w} p_{g}\left(\frac{r}{w}\right) p_{w}(w) d w=\int_{0}^{\infty} \frac{1}{w}\left(s_{g} p_{\text {Rice }}\left(\frac{r}{w}\right)+s_{b} p_{\text {Suzuki }}\left(\frac{r}{w}\right)\right) p_{w}(w) d w \\
& =s_{g} \int_{0}^{\infty} \frac{1}{w} p_{\text {Rice }}\left(\frac{r}{w}\right) p_{w}(w) d w+s_{b} \int_{0}^{\infty} \frac{1}{w} p_{\text {Suzuki }}\left(\frac{r}{w}\right) p_{w}(w) d w
\end{aligned}
$$

\section{Implementation of the Ka-Band Satellite Channel Comprehensive Model}

According to the Ka-band mobile satellite channel propagation characteristics, the simulation model of Ka-band mobile satellite channel comprehensive model is established by Simulink simulation software. This simulation model introduces the multiplicative fading module due to the weather impairments and the two-state fading module due to the mobile environment, and the two modules are independent of each other. The realizations of the two modules are all based on a number of uncorrelated colored Gaussian random processes. In this paper, the method of sun-of-sinusoids (SOS) is employed for designing colored Gaussian random processes[7]. The main idea of the SOS principle is to approximate colored Gaussian processes by a finite sum of sinusoidal waveforms. The deterministic Gaussian process is given by

$$
\mu_{i}(t)=\sum_{n=1}^{N_{i}} c_{i, n} \cos \left(2 \pi f_{i, n} t+\theta_{i, n}\right) \quad i=1,2,3
$$

where $N_{i}$ denotes the number of sinusoids and $N_{1}=N_{2}=8, N_{2}=9$. The parameters $c_{i, n}, f_{i, n}$ and $\theta_{i, n}$ are the simulation model parameters called as the Doppler gains, the Doppler frequencies, and the Doppler phases, respectively. They are defined as 


$$
\begin{aligned}
c_{i, n} & =\sigma \sqrt{\frac{2}{N_{i}}} \quad i=1,2 \\
f_{i, n} & =f_{\max } \sin \left[\frac{\pi}{2 N_{i}}\left(n-\frac{1}{2}\right)\right] \\
\theta_{i, n} & =\frac{2 \pi n}{N_{i}+1}
\end{aligned}
$$

where $\sigma$ denotes the standard deviation, $f_{\max }$ is the maximum Doppler frequency.

In the study of the satellite channel characteristics, Rayleigh process, Rician process, and Lognormal process all can be achieved by the colored Gaussian process. The Simulink diagrams are shown in Fig.1 and Fig.2.

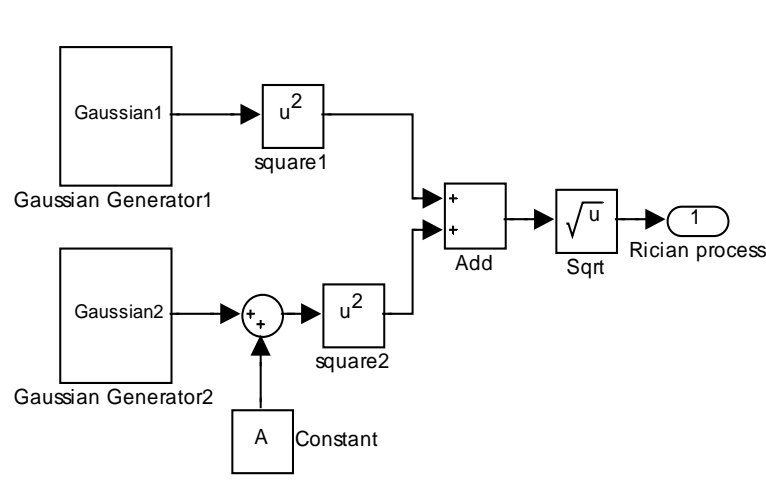

Fig. 1 Simulink of Rician process

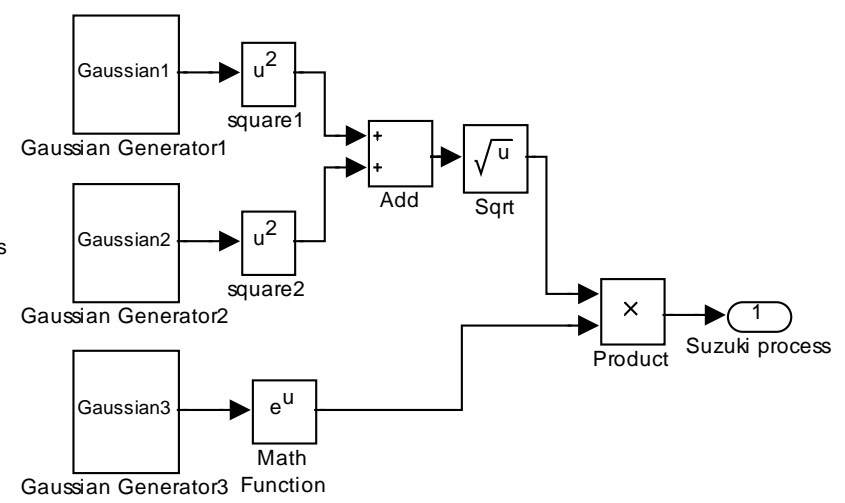

Fig. 2 Simulink of Suzuki process

The simulation model of Ka-band mobile satellite communication system is shown in Fig.3. The data sources generate the binary signal, the binary signal is modulated and put into the channel. The signal is influenced by the weather conditions, the mobile environment, and the additive white Gaussian noise in the channel. And then it is demodulated and output. Finally, the bit error rate (BER) performance is analyzed by the BER tools.

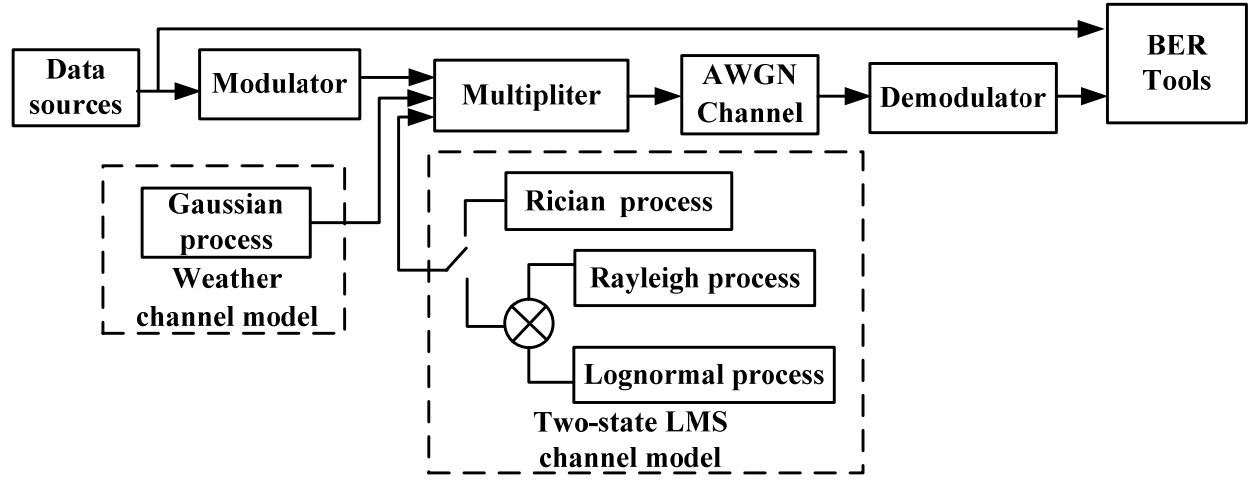

Fig. 3 Simulation model of Ka-band mobile satellite communication system

\section{Simulation analysis}

To verify the feasibility of the proposed Ka-band mobile satellite comprehensive model, the BER curves in different channel environment are presented. Simulation results are shown in Fig.4.In Fig.4, the BER performances under the four main weather conditions are shown in Fig.4(a), Fig.4(b) shows the BER performance of the two-state channel, which is affected by the mobile environment, and the BER performances of the comprehensive model under different weather conditions are shown in Fig.4(c). The BER curves show that the BER performance of the two-state channel is between the good-state and the bad-state, it is consistent with the propagation characteristics of the Ka-band mobile satellite channel. The simulation results show that the signal attenuation is different with 
different weather conditions, and the signal attenuation is most serious in thunder rain. From Fig.4 , the influence of weather impairments is smaller but can't be ignored.

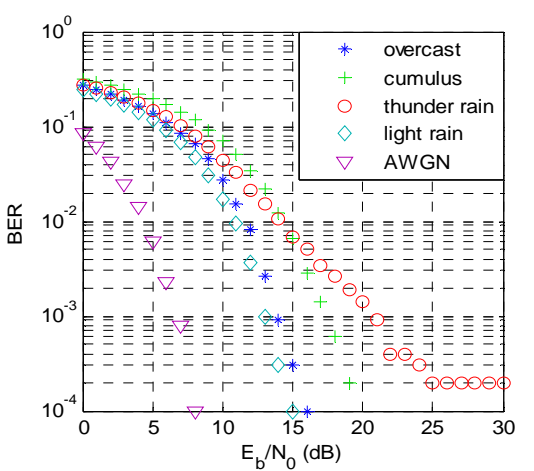

(a) BER under weather conditions

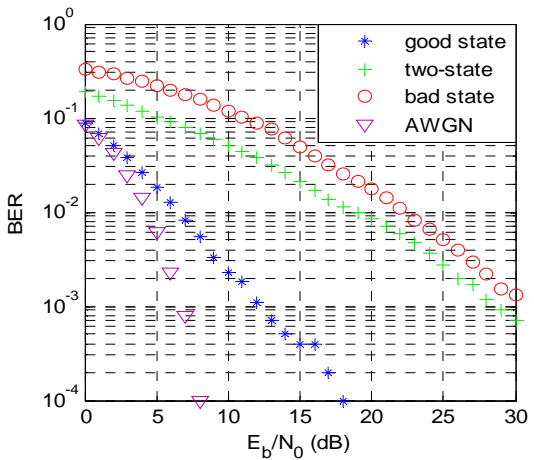

(b) BER of two-state channel Fig.4 Simulation results

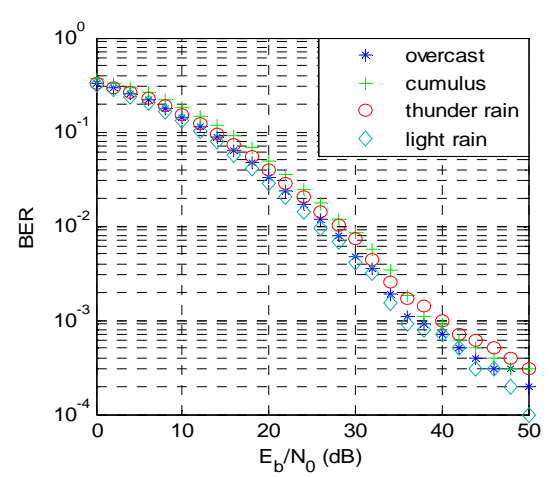

(c) BER of comprehensive model

\section{Summary}

In the Ka-band satellite channel comprehensive model, the weather impairments and the mobile environment are considered simultaneously to overcome the disadvantages of the previous static channel models. The theoretical analysis and simulated results show that the proposed model is appropriate to describe the propagation characteristics of the Ka-band satellite channel.

\section{Acknowledgement}

This work is supported by the National Natural Science Foundation of China(Grant No. 51405241, 11504176,61371131), the Major Project of Nature Science Foundation of Higher Education Institution of Jiangsu Province, China(Grant No.13KJA510001), Jiangsu Scientific Research Achievements in Industrialization Project, China(JHB 2012-9), and JiangsuProvince, the 2015 Annual General University Graduate Students Practice Innovation Program(SJLX15_0398).

\section{References}

[1] Lutz E. Modeling of the land mobile satellite communications channel[C]. IEEE-APS Topical Conference on Antennas and Propagation in Wireless Communications(APWC), Torino, 2013,199-202.

[2] Lin Shuxian, Zhu Lidong, Guo Yantao, et al. Distribution characteristics and performance simulations of rain attenuation at Ka band for satellite communications[C]. 5th Gobal Symposium on Millimeter Waves(GSMM), Harbin, 2012, 579-582.

[3] Pan Chengsheng, Li Huafang, Liu Chunling. Statistical analysis of bit error in Ka band mobile satellite channel [J]. Journal of Computer Applications, 2012, 32(8):2137-2140.

[4] Zain A F M, Albendag A A M. Improving ITU-R rain attenuation model for HAPS earth-space link[C]. IEEE International Conference on Space Science and Communication(IconSpace), Melaka, 2013, 56-59.

[5] Louis J. Ippolito Jr. Satellite communications systems engineering[M]. Sun Baosheng, translated. Beijing: Nation Defense Industry Press, 2012, 109-111.

[6] Yang M C, Meng F Y, Shi S, et al. Markov chain based two-state satellite mobile channel model[C]. 2011 IEEE 73rd Vehicular Technology Conference (VTC Spring), Yokohama, 2011, 1-5.

[7] Patzoid M, Cheng-Xiang Wang, Hogstad B O. Two new sum-of-sinusoids-based methods for the efficient generation of multiple uncorrelated Rayleigh fading waveforms[J]. IEEE Transactions on Wireless Communications. 2009, 8(6):3122-3131. 